\title{
Semi-dynamic Modeling of Shell Formation for V4334 Sgr (Sakurai): An Online Probe for Early PNe Evolution?
}

\author{
Stefan Kimeswenger \\ Institut für Astrophysik, Leopold-Franzens Universität Innsbruck, \\ Technikerstraße 25, A-6020 Innsbruck, AUSTRIA
}

\begin{abstract}
The central star V4334 Sgr (Sakurai's Nova) of the planetary nebula PN G010.4+04.4 underwent in 1995-1996 the rare event of a very late helium flash shell burning. The rapid formation of a dust shell allows a precise model, basing on the dust formation history instead of the commonly used average dust formation rate and mass loss. The model applies a complete dust grain size distribution, and transiently heated grains. Only the fitting of the visual light curve gives already the complete spectral energy distribution (SED) model. No adjustment with the infrared data points is needed.
\end{abstract}

The central star V4334 Sgr (Sakurai's Nova) of the planetary nebula PN G010.4+04.4 underwent in 1995-1996 the rare event of a very late helium flash shell burning (Iben et al. 1983). It is only one of two such events in the era of modern astronomy (the second event was V605 Aql = Nova Aql 1919; for a review see Clayton and de Marco 1997). Other prominent objects of that type originated from events several thousands of years ago (for A30: Borkowski et al. 1984; for A78: Kimeswenger et al. 1998). Hence, only snapshot models can be designed for those objects. V4334 Sgr allows for the first time a consideration of the dynamic formation of the dust shell from the beginning. The rapid formation of a dust shell, discovered in March 1997 (Kimeswenger et al. 1997), started in the end of 1996. Several snapshot models of this object were published (Kerber et al. 1998, 1999; Kipper 1999). They suffer from the fact that they allow only the use of an average dust formation rate and mass loss history. Thus, they are unable to describe the visual photometry and provide only crude estimates for the density distribution and for the mass of the shell. Such shells also do not describe the NIR/MIR SED well (Kerber et al. 1999).

I am using here the visual light curve to determine the variations of the dust formation rate. The model applies a complete dust grain size distribution and transiently heated grains (Koller \& Kimeswenger 2000, 2001). The visual light curve in I and V was used to determine the "global" property like the general trend in effective temperature and luminosity of the star. A distance of $3 \mathrm{kpc}$ to the object was assumed (Kimeswenger 2001). The "dips" in the light curve were used to derive the quasi periodic outbursts of the dust formation rate. This dust formation rate directly leads to a model of the dynamic evolution of the density profile. This density profile was used to calculate a spectral energy distribution (SED) at different epochs of ISO observations (Kerber et al. 1999), which turned out to be sufficient without further fitting to the data points! The overall dust formation rate shows in 1997 a linear relation with the luminosity 
and has outbursts on timescales of 50 to 60 days lasting typically 15 days. As of end of 1997, the dust shell mass has grown to $2.510^{-7} \mathrm{M}_{\odot}$. The input from the fitting of the visual light curve gives already a complete SED model.
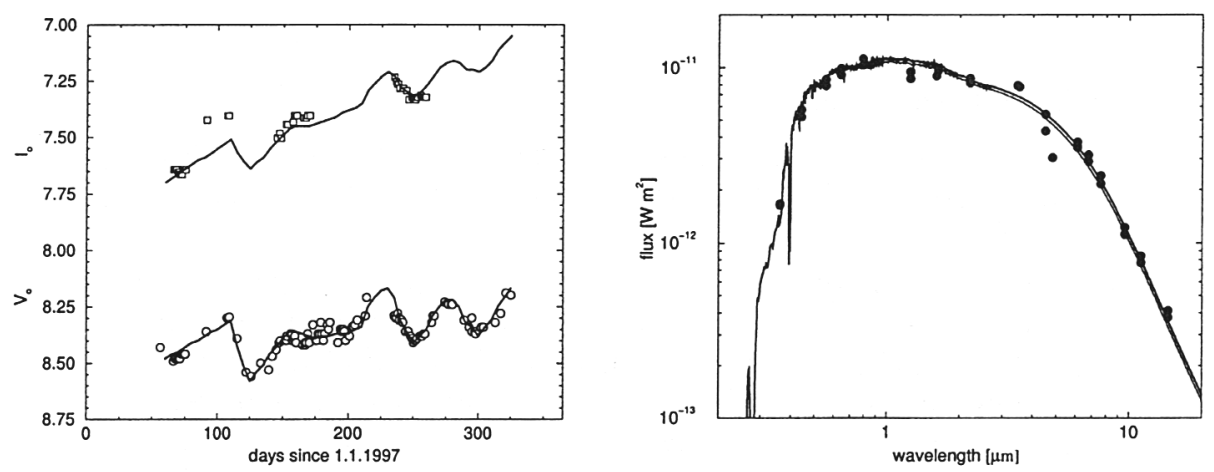

Figure 1. The light curve used to derive the mass loss history profile (left) and the resulting SED for one of the ISO observation epochs (September 1997; right).

Even if the very late helium flash occurs at a very late stage of the $\mathrm{PNe}$ evolution, the online insight into the rapid processes of forming a shell gives us information about the birth of "normal" PNe. The abundance of heavy elements is unusually high in those shells. This results in a higher dust formation rate. Thus a significant speeding of the evolution occurs. Within a few years this allows, in spite of all differences in the initial nature, to obtain insights to those processes, which could be worked out only in a laborious way.

\section{References}

Borkowski, K.J., Harrington, J.P., Blair, W.P., \& Bregman, J.D. 1994, ApJ, 235, 722 Clayton, G.C., \& deMarco, O. 1997, AJ, 114, 2679

Iben, I., Kaler, J.B., Truran, J.W., \& Renzini, R. 1983, ApJ, 264, 605

Kerber, F., Gratl, H., Kimeswenger, S., \& Roth, M. 1998, Ap\&SS, 255, 279

Kerber, F., Blommaert, J.A.D.L., Groenewegen, M.A.T., Kimeswenger, S., Käufl, H.-U., \& Asplund, M., 1999, A\&A, 350, L27

Kimeswenger, S. 2001, Ap\&SS, in press, astro-ph/0105119

Kimeswenger, S., Gratl, H., Kerber, F., et al. 1997, IAUC 6608

Kimeswenger, S., Kerber, F., \& Weinberger, R. 1998, MNRAS, 296, 614

Kipper, T. 1999, Baltic Astronomy, 8, 483

Koller, J., \& Kimeswenger, S. 2000, in ASP Conf. Ser. Vol. 196, Thermal Emission Spectroscopy and Analysis of Dust, Disks, and Regoliths, ed. M.L. Sitko, A.L. Sprague \& D.K. Lynch (San Francisco: ASP), 23

Koller, J., \& Kimeswenger, S. 2001, ApJ, 559, 419 